\title{
I = Parma, Biblioteca Palatina, 154 (HH. IX 23)
}

Costantinopoli, XIV sec.

ff. A, I-IX, 2-115, X, A'; sul f. Av è segnato il num. 319 e Eur. Sec. 15º; sul f. II è ripor-

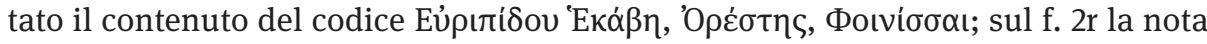
di possesso depennata di Niccolò Michelozzi (Nicolai Michelotii Florentini Liber); num. fogli recente a penna nel marg. sup. est.; cart.; fil. ff. 3, 5, 8, 10, 12, 19, 21, 23 , 24, 26, 29, 31, 32, 34, 37, 40, 42, 45, 47, 48, 50, 52, 54, 57, 59, 60, 63, 64, 67, 69, 71, 72, 74, 77, 79, 81, 85, 86, 89, 90, 93, 95, 96, 98, 101, 102, 105, 106, 109, 111, 112, 114 simile a cercle (M/Tr nr. 2160, 1367), ff. 14, 17 lettres (M/Tr nr. 5125, 1369); num. fasc. visibile in alcuni casi nel marg. est. del recto del primo foglio o nel marg. inf. est. del verso dell'ultimo foglio dei fascicoli, numerati da $\beta^{\prime}$ (f. 12) a $\alpha^{\prime}$ (f. 91v); $1^{10}(10), 2-14^{8}$ (115); mm $225 \times 145=25$ // 172 // $30 \times 17$ // 60 // 11 // 44 // 12; una colonna, 1l. 20, unità di rigatura $\mathrm{mm} 8$; rigatura tracciata a secco sul recto di ogni foglio, tipo S/L 30D1 (Muz 2-21/0-0/J) (f. 30r); mm $225 \times 145=13 / / 188 / / 22 \times 15 / / 110 / / 15$, a piena pagina, 1l. 36 , unità di rigatura $\mathrm{mm} 5$, rigatura tracciata a secco, tipo S/L 10D1n (Muz 1-2/0/0/J) (ff. 2v, 33r, 73v). Legatura in marocchino rosso su cartone; sul piatto anteriore è impressa la scritta crisografa Bibliothecae Palatinae Regiae Parmensis accompagnata da tre gigli borbonici; sul dorso in alto, sempre in lettere dorate, compare la scritta Euripid Mss Chart, in basso è incollato un foglio con la segnatura del codice (Parm. 154).

\section{Scrittura}

Il codice è stato vergato da un'unica mano: <Isacco Argiro (Bianconi 2008, 361; Mondrain 2007 [2008], 169)> (ff. 2r-144v).

\section{Decorazione}

Decorazione minima, con rubriche in inchiostro rosso.

\section{Contenuto}

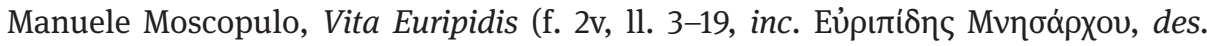

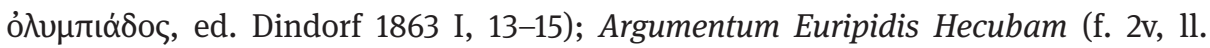

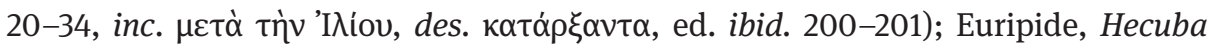

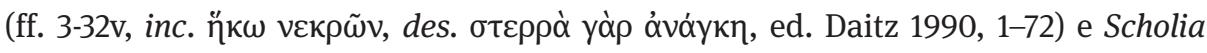


et scholia metrica in Euripidis Hecubam (ff. 3r-32v, ed. Smith 1977, 1-38; ed. Schartau

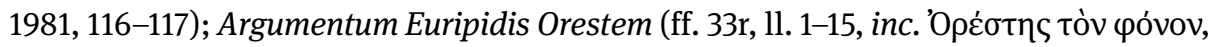

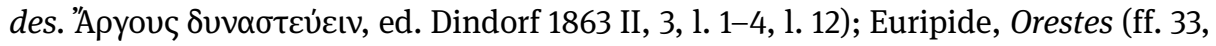

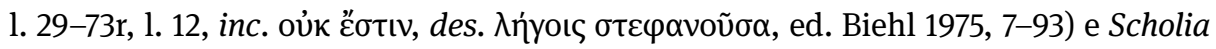

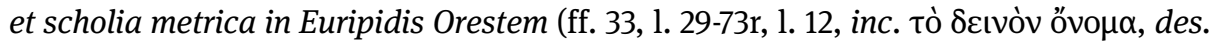



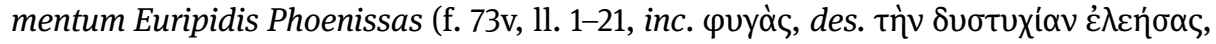

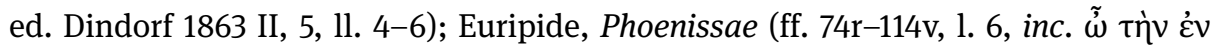

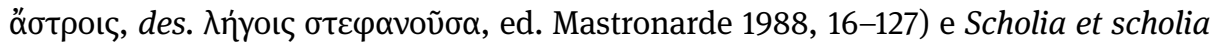

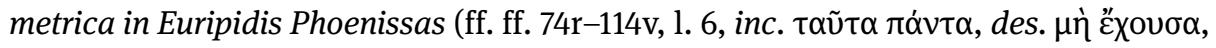
ed. Smith 1977; ed. Schartau 1981, 226).

\section{Relazioni stemmatiche}

Le tragedie euripidee sono corredate da commenti metrici e scolii esegetici peculiari; si tratta di un Mischkommentar, che accosta ai commenti e agli scolii di Moscopulo e di Triclinio un numero considerevole di annotazioni originali da riferire ad Argiro (Schartau 1981, 238-239; Bianconi 2008, 361, n. 71). L'unico altro manoscritto recante questi scolii, e per questo considerato gemello del codice di Parma, è il Mut. a.U.9.22, copiato da Andronico Callisto (Schartau 1981, 238-239).

\section{Bibliografia}

\section{Cataloghi}

Eleuteri (1993) 39-41; Martini (1893) 175-179.

\section{Edizioni}

Biehl (1975); Daitz (1990); Dindorf (1863); Mastronarde (1988); Schartau (1981); Smith (1977).

\section{Codicologia e paleografia}

Bianconi (2008) 361 e n. 77; Bianconi (2010) 492-493; Mondrain (2006-2007, 2008) 52-54; Mondrain (2007) [2008] 169; Muratore (2009) I, 182; II, 128. 


\section{Studi}

Diggle (1991) 10, 103-110; Mastronarde/Bremer (1982) 15, 30, 137-140, 146, 164-166; Smith (1974) 414-415; Smith (1977) IX-XII, XIV-XxIV; Smith (1981-1982) 253-256.

\section{Riproduzioni}

Bianconi (2008) tav. 15; Eleuteri (1993) tav. XVII; Smith (1977) tav. 6. 